\title{
Geometric representation of spin correlations and applications to ultracold systems
}

\author{
Rick Mukherjee, Anthony E. Mirasola, Jacob Hollingsworth," Ian G. White, and Kaden R. A. Hazzard \\ Department of Physics and Astronomy, Rice University, Houston, Texas 77005, USA \\ and Rice Center for Quantum Materials, Rice University, Houston, Texas 77005, USA
}

(Received 23 December 2016; revised manuscript received 6 March 2018; published 6 April 2018)

\begin{abstract}
We provide a one-to-one map between the spin correlations and certain three-dimensional shapes, analogous to the map between single spins and Bloch vectors, and demonstrate its utility. Much as one can reason geometrically about dynamics using a Bloch vector-e.g., a magnetic field causes it to precess and dissipation causes it to shrink - one can reason similarly about the shapes we use to visualize correlations. This visualization demonstrates its usefulness by unveiling the hidden structure in the correlations. For example, seemingly complex correlation dynamics can be described as simple motions of the shapes. We demonstrate the simplicity of the dynamics, which is obscured in conventional analyses, by analyzing several physical systems of relevance to cold atoms.
\end{abstract}

DOI: 10.1103/PhysRevA.97.043606

\section{INTRODUCTION}

Correlations play an important role in all branches of science. They determine the distribution of galaxies in cosmology [1], reveal the complex structure of molecules and proteins in chemistry and biology [2,3], are invaluable in quantum sensing and computing in the form of quantum entanglement [4-10], and are used to test fundamental predictions of quantum chromodynamics (QCD) about entangled quark pairs [11,12]. In particular, correlations are of fundamental interest in manybody physics as they characterize phases in condensed matter [13-15] and in ultracold matter [16-23].

However, even the simplest correlations in many-body systems have considerable complexity. For example, consider correlations between two spin- $1 / 2 \mathrm{~s}$, as illustrated in Fig. 1(a). The correlations between each pair of spins $i$ and $j$ are described by $\left\langle\sigma_{i}^{\alpha} \sigma_{j}^{\beta}\right\rangle$, with $\alpha, \beta \in\{x, y, z\}$, and therefore require nine components to specify. Furthermore, as Fig. 1(b) illustrates, the behavior of these components can be complicated and seemingly structureless. Similarly, other standard visualizations such as density matrix tomography plots [Fig. 1(c)] fail to reveal any obvious structure.

In this paper, we provide a method that encodes all of the correlation components holistically in a geometric object. We demonstrate that seemingly complicated, structureless dynamics is in fact simple motions of these geometric shapes for several examples of many-body systems. For example, our visualization in Fig. 1(d) reveals the superficially complicated dynamics of Figs. 1(b) and 1(c) to be a simple growth and rotation of an object in the shape of a clover. Our examples are drawn from recent experiments in ultracold matter, including lattice fermions [24,25], Rydberg atoms [26-29], molecules [30-32], and trapped ions [33-37], but the visualization techniques are completely general.

Our work is not the first to consider geometric visualizations of spin correlations, but builds on the useful tools in

*Current address: University of California at Irvine, Irvine CA 02697.
Refs. [38-60]. Although we provide different methods (see Sec. III) to interpret the shapes, such as how to read off correlations from the shapes, our constructions is in essence equivalent to that in Refs. [53-56]. However, these prior studies considered quantum states chosen at random or for illustrative purposes. In contrast, we apply these tools to two-particle correlations in real many-body systems. In doing so, we show that not only are the shapes a compact way to summarize the spin components, but also that physical phenomena which appear complicated and mysterious when considering their components are simple, easy-to-describe motions when visualized geometrically.

This paper first presents, in Sec. II, the prescription for generating the visualizations. Section III discusses how to interpret these correlation matrix visualizations (CMVs), whose size and color convey the magnitude and sign of the correlations. Its main goal is to give a feeling for the CMVs and how to use them by first applying them to simple example states, where the states' properties are familiar from other methods. It shows how these familiar properties appear in the CMVs and gives a general prescription for "reading" them. Section IV applies our visualization techniques to different scenarios where the correlations are much less obvious and unveils a structure that is not apparent in these other representations of correlations. We visualize correlations of systems, both in nonequilibrium and equilibrium, that are relevant to ultracold matter. It shows that in all cases, the visualizations provide an especially simple representation of the behavior. Although we focus on two-particle correlations that are symmetric, our method can be generalized to include more spins and allow asymmetric correlations, as briefly explained in Appendices A and B.

\section{RECIPE FOR GENERATING A CORRELATION MATRIX VISUAL (CMV)}

We are interested in visualizing the two-point connected spin correlations. Accordingly, we define the one-spin and twospin observables. The single spin Bloch vector is

$$
\mathbf{b}_{i}=\left(b_{i}^{x}, b_{i}^{y}, b_{i}^{z}\right)=\left(\left\langle\sigma_{i}^{x}\right\rangle,\left\langle\sigma_{i}^{y}\right\rangle,\left\langle\sigma_{i}^{z}\right\rangle\right)=\left\langle\sigma_{i}\right\rangle,
$$




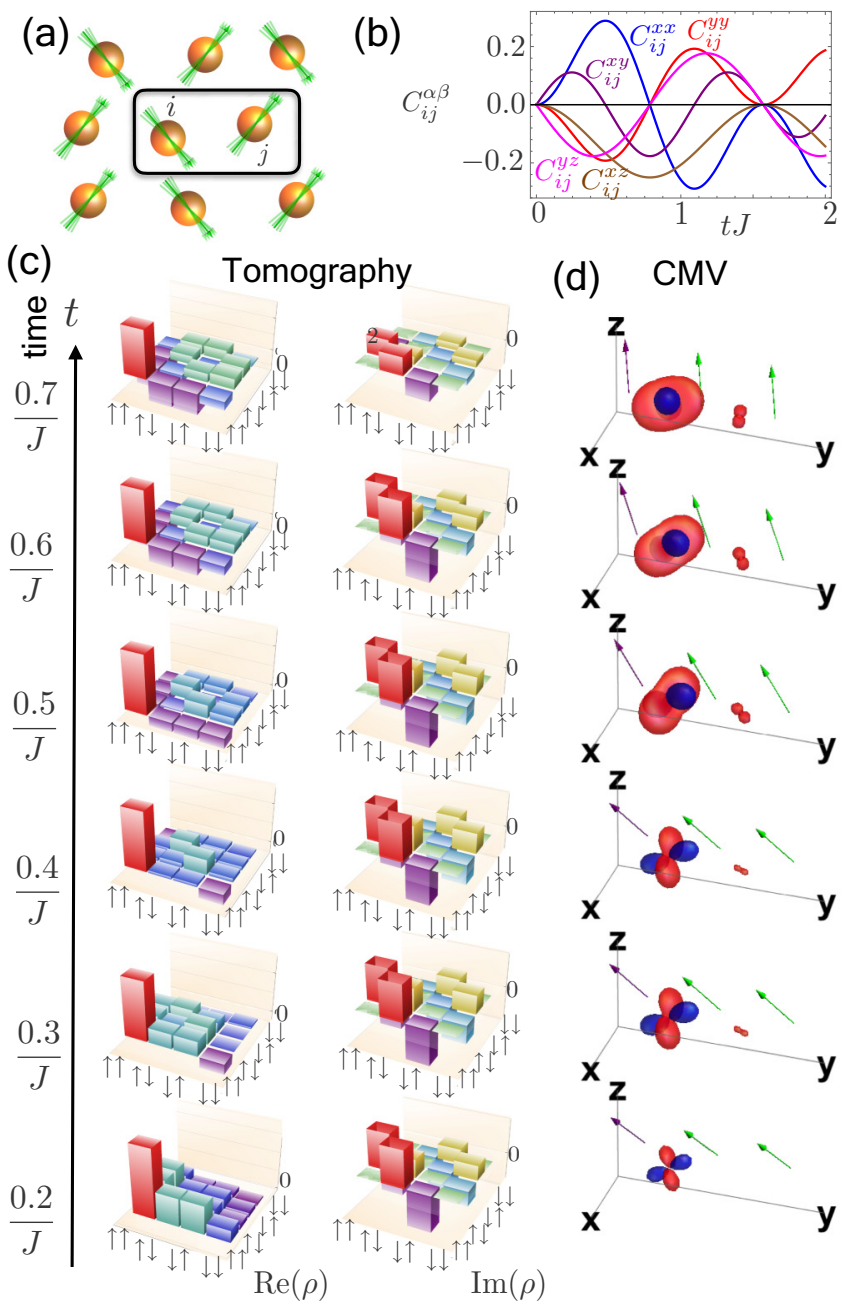

FIG. 1. Unveiling of hidden structure in correlations by our visualization method. (a) Correlations in a many-body system of spin-1/2s. Green arrows are the Bloch vectors of each spin. (b) Complicated evolution of spin correlation components $C_{i j}^{\alpha \beta}$ vs time with $\alpha, \beta=x, y, z$ and $i, j$ index the spins. (This example is calculated for Ising dynamics, discussed in Sec. IV A.) (c) Tomographic representation of the two-spin density matrix for the same dynamics, showing $\langle a b|\rho| c d\rangle$ for $\{a, b, c, d\} \in\{\uparrow, \downarrow\}$. (d) Our visualization method [correlation matrix visualization (CMV)] applied to the same dynamics reveals that the complicated components hide a very simple dynamics: a "clover" shape grows in a plane perpendicular to the Bloch vector and rotates.

where $i$ labels the particle and $\sigma^{x, y, z}$ are the Pauli operators. The correlations between a pair of spins $i$ and $j$ are

$$
c_{i j}=\left(\begin{array}{ccc}
c_{i j}^{x x} & c_{i j}^{x y} & c_{i j}^{x z} \\
c_{i j}^{y x} & c_{i j}^{y y} & c_{i j}^{y z} \\
c_{i j}^{z x} & c_{i j}^{z y} & c_{i j}^{z z}
\end{array}\right),
$$

where $c_{i j}^{\mu \nu}=\left\langle\sigma_{i}^{\mu} \sigma_{j}^{\nu}\right\rangle$ and $\mu, \nu \in\{x, y, z\}$. The connected correlations are

$$
C_{i j}^{\mu \nu}=c_{i j}^{\mu \nu}-b_{i}^{\mu} b_{j}^{v} .
$$

Matrix elements $C_{i j}^{\mu \nu}$ are real for $i \neq j$. To obtain a geometric visualization of the correlation matrix $C_{i j}$, our first step is to relate it to the quadratic form

$$
Q\left(C_{i j}, \mathbf{r}\right)=\mathbf{r}^{T} C_{i j} \mathbf{r}=\sum_{\mu, v \in\{x, y, z\}} C_{i j}^{\mu v} r^{\mu} r^{\nu},
$$

where $\mathbf{r}=(x, y, z)$. One can visualize a quadratic form through its level sets (surfaces of constant value). This visualization has the drawback that the level sets of some quadratic forms are not compact (e.g., a hyperboloid) and thus not easily represented in a finite space. To associate a finite object with each quadratic form, we plot the level sets of

$$
Q_{f}\left(C_{i j}, \mathbf{r}\right)=\frac{Q\left(C_{i j}, \mathbf{r}\right)}{\left(1+r^{2}\right)^{3 / 2}}
$$

The choice of denominator is fairly arbitrary and other choices, such as multiplying $Q$ by $e^{-r}$, could be made. We find that the choice in Eq. (5) gives a large dynamic range across which it keeps the figure size roughly proportional to the size of the correlations (Appendix D). We plot a level set (in red) where $Q_{f}$ attains a given positive value $P$, and another (in blue) where it attains $-P$. We typically choose $P=0.01$. We refer to this picture of level sets as a correlation matrix visualization (CMV).

This method of visualization is sensitive only to the symmetric part of the correlation matrix. Many important correlation matrices are symmetric; for example, correlation matrices are symmetric in translationally invariant systems, the case we consider in this paper. For such symmetric correlations, $Q_{i j}$ is in one-to-one correspondence with $C_{i j}$. In this case, the CMVs are equivalent to the correlation matrices. Generalizations of our scheme to visualize $N$-body correlations are discussed in Appendices $\mathrm{B}$ and $\mathrm{C}$, and to visualize asymmetric correlation matrices in Appendix A. Although we work primarily with connected correlations $C_{i j}$, our scheme works just as well for correlations $c_{i j}$.

Furthermore, there is a one-to-one correspondence between the connected correlation matrix together with the Bloch vectors and the reduced two-spin density matrix $\rho_{i j}$. This can be written in terms of the Pauli matrices as

$$
\rho_{i j}=\frac{1}{4} \sum_{\alpha, \beta=0}^{3} S_{i j}^{\alpha \beta} \sigma_{i}^{\alpha} \otimes \sigma_{j}^{\beta},
$$

where $\sigma^{\alpha} \in\left\{11, \sigma^{x}, \sigma^{y}, \sigma^{z}\right\}$ and $S_{i j}^{\alpha \beta}=\operatorname{Tr}\left(\rho_{i j} \sigma_{i}^{\alpha} \otimes \sigma_{j}^{\beta}\right)$, giving

$$
S_{i j}=\left(\begin{array}{cccc}
1 & b_{i}^{x} & b_{i}^{y} & b_{i}^{z} \\
b_{j}^{x} & c_{i j}^{x x} & c_{i j}^{x y} & c_{i j}^{x z} \\
b_{j}^{y} & c_{i j}^{y x} & c_{i j}^{y y} & c_{i j}^{y z} \\
b_{j}^{z} & c_{i j}^{z x} & c_{i j}^{z y} & c_{i j}^{z z}
\end{array}\right) .
$$

Consequently, these three things are equivalent (still under the assumption that the correlation matrix is symmetric): (1) the CMV along with the Bloch vectors, (2) the correlation matrix $C_{i j}$ along with the Bloch vectors, and (3) the two-spin density matrix. We will see that examining the CMVs makes apparent physics that is hidden in the latter two representations.

\section{HOW TO READ A CMV}

In this section, we discuss two essential aspects: how to read a CMV and how to characterize it. One of the biggest 


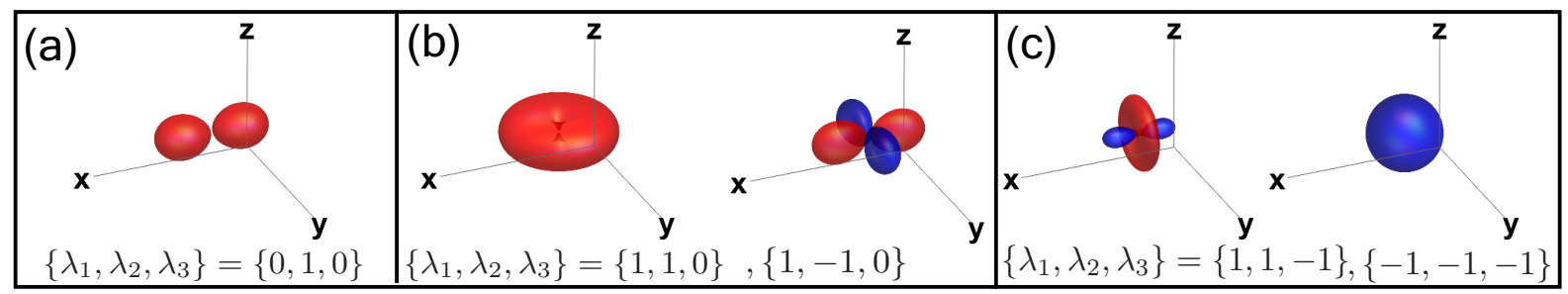

FIG. 2. CMV shapes: The shape of the CMV is determined by the set of nonzero eigenvalues $\left\{\lambda_{1}, \lambda_{2}, \lambda_{3}\right\}$ of the correlation matrix $C_{i j}$ and their relative signs. Positive (negative) correlations are depicted as red (blue) CMV surfaces. (a) CMVs with a single nonzero eigenvalue (rank-1 CMVs) are dumbbells. (b) Rank-2 CMVs occur in two possible topologies. If the eigenvalues' signs are equal, one obtains a "disk" of either color (left), while if their signs are opposite, one obtains a "clover" shape (right). (c) Rank-3 CMVs occur in two possible topologies. If all of the eigenvalues' signs are equal, the CMV is a sphere; if one eigenvalue has a sign opposite the other two, one obtains a "wheel-and-axle" shape.

advantages of our visualization is that one can deduce the magnitude of the correlations along any direction directly from the size of the CMV in that direction. The connected correlations between a pair of spins along a direction $\mathbf{e}$ is given as

$$
\begin{aligned}
& \left\langle\left(\boldsymbol{\sigma}_{i} \cdot \mathbf{e}\right)\left(\boldsymbol{\sigma}_{j} \cdot \mathbf{e}\right)\right\rangle-\left\langle\boldsymbol{\sigma}_{i} \cdot \mathbf{e}\right\rangle\left\langle\boldsymbol{\sigma}_{j} \cdot \mathbf{e}\right\rangle \\
& =\sum_{\mu, v \in\{x, y, z\}}\left\langle\left(\sigma_{i}^{\mu} e^{\mu}\right)\left(\sigma_{j}^{v} e^{\nu}\right)\right\rangle-\left\langle\sigma_{i}^{\mu} e^{\mu}\right\rangle\left\langle\sigma_{j}^{v} e^{\nu}\right\rangle \\
& =\mathbf{e}^{T} C_{i j}^{\mu \nu} \mathbf{e} \\
& =Q\left(C_{i j}, \mathbf{e}\right) .
\end{aligned}
$$

When $Q\left(C_{i j}, \mathbf{e}\right)$ is large in absolute value, the CMV (with the appropriate sign) will be large in this direction as well (see Appendix D). Consequently, one can read off the size of correlations along a certain direction by the size of the CMV in that direction.

Using this geometric interpretation, we now proceed to interpret the CMVs in Fig. 2. For example, consider the dumbbell-like CMV in Fig. 2(a) lying along the $x$ axis. This CMV has its largest correlations along a line parallel to the $x$ axis which passes through its center. More specifically, $C^{x x} \neq$ 0 while $C^{y y}=C^{z z}=0$. Similarly, consider the clover-shaped CMV in Fig. 2(b). Visually one can infer that $C^{x x}, C^{y y} \neq 0$, but the correlation along a line $45^{\circ}$ rotated from $x$ in $x y$ plane is zero. Finally, the sphere in Fig. 2(c) indicates that the correlation components are rotationally symmetric. Also note that we have chosen the origin arbitrarily in order to make the CMVs easier to see.

The shape and color of a CMV can be related to the eigenvalues of the correlation matrix. By shape we mean geometrical properties of the CMV that are invariant under rotation. Let us therefore consider a rotation matrix $R$ that rotates our original CMV such that $\mathbf{r}^{\prime}=R \mathbf{r}$. Then,

$$
Q\left(C_{i j}, \mathbf{r}^{\prime}\right)=(R \mathbf{r})^{T} C_{i j}(R \mathbf{r})=\mathbf{r}^{T}\left(R^{T} C_{i j} R\right) \mathbf{r}=Q\left(C_{i j}^{\prime}, \mathbf{r}\right),
$$

where we define $C_{i j}^{\prime}=R^{T} C_{i j} R$. In other words, two CMVs that are related by a rotation $R$ are associated with correlation matrices $C_{1}$ and $C_{2}$ that are similar to each other under $R$ (and thus have the same eigenvalues). The converse is also true. That is, if we have two correlation matrices that are related by rotations, then they are represented by CMVs that have the same shape, but different orientations. As an example, the Bell states $\left(\left|\phi^{ \pm}\right\rangle,\left|\psi^{+}\right\rangle\right)$, which are shown in Fig. 3(a), illustrate the physical significance of this rotational equivalence. Each of these CMVs can be rotated to any other because these three Bell states are equivalent under global rotations. The symmetry of each CMV and their relation to each other simply reflect the symmetry of the corresponding wave functions.

Although the topology and colors are the most visually apparent features of the CMV, they do not uniquely describe a state. Other aspects such as the size of the CMV and the corresponding Bloch vectors are also needed to specify even the twospin density matrix. In addition, the CMV representation for a two-spin correlation of a many-body system does not uniquely specify the many-body state. For example, two distinct threespin states can have identical pairwise correlations, but are distinguished by their three-body correlations. We see this when we compare the CMV of the two-particle correlation for a three-particle GHZ state with that of the mixed states in Figs. 3(b) and 3(d), respectively, where both have the dumbbell topology but differ only in size. The same is true when we

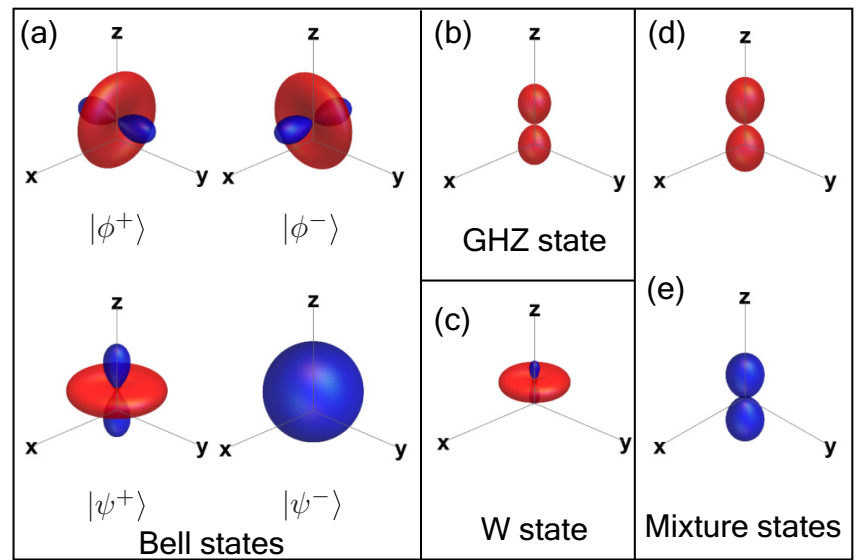

FIG. 3. CMVs for prototypical states from the density matrix, $\rho=|\Psi\rangle\langle\Psi|$ : (a) Bell states $|\Psi\rangle=\left|\phi^{ \pm}\right\rangle=(|\downarrow \downarrow\rangle \pm|\uparrow \uparrow\rangle) / \sqrt{2}$, Bell states $|\Psi\rangle=\left|\psi^{ \pm}\right\rangle=(|\downarrow \uparrow\rangle \pm|\uparrow \downarrow\rangle) / \sqrt{2}$; (b) Two-spin correlations in a three-spin GHZ state, $|\Psi\rangle=(|\downarrow \downarrow \downarrow\rangle+|\uparrow \uparrow \uparrow\rangle) / \sqrt{2}$; (c) Two-spin correlations in a three-spin $W$ state, $|\Psi\rangle=$ $(|\downarrow \downarrow \uparrow\rangle+|\downarrow \uparrow \downarrow\rangle+|\uparrow \downarrow \downarrow\rangle) / \sqrt{3}$. CMVs for simple mixtures of product states: (d) $\rho=(|\downarrow \downarrow\rangle\langle\downarrow \downarrow|+| \uparrow \uparrow\rangle\langle\uparrow \uparrow|) / 2$ and (e) $\rho=$ $(|\downarrow \uparrow\rangle\langle\downarrow \uparrow|+| \uparrow \downarrow\rangle\langle\uparrow \downarrow|) / 2$. 
compare the three Bell states $\left(\left|\phi^{ \pm}\right\rangle,\left|\psi^{+}\right\rangle\right)$with the $W$ state. The Bloch vectors are not explicitly shown in Fig. 3. They are zero for all the states except for the $W$ state.

\section{CMVS FOR PHYSICALLY REALIZABLE SPIN MODELS}

Here we calculate and describe the CMVs that arise in prototypical many-body systems. We focus on a few examples that are motivated by experiments on ultracold matter. In particular, we consider the nonequilibrium dynamics in the Ising model evolved from a product-state ferromagnet (Secs. IV A and IV B), the dynamics of the Fermi-Hubbard model (Sec. IV C) evolved from a product-state canted antiferromagnet, and the equilibrium correlations of the transverse-field Ising model across its phase diagram (Sec. IV D). All of these models have been realized in ongoing experiments, as briefly described in their respective sections. Conveniently, they also can be solved exactly. We focus on one-dimensional systems with nearest-neighbor (NN) couplings for simplicity. In all cases, we find that although the correlations can be complicated, the CMVs behave in a simple manner.

\section{A. Coherent Ising model}

The Ising model has been extensively studied in the context of ferromagnetism and phase transitions [13], and has been applied to study cooperative behavior in fields as far removed as biology [61]. In ultracold matter, there are many proposals and realizations of Ising Hamiltonians, for example, using trapped ions [33-35,62], dipolar molecules [30-32,63-67] and atoms [68,69], Rydberg atoms [23,26-29,70-73], and tilted Bose-Hubbard systems [74,75]. Reference [76] overviews these systems and others in which the Ising model can and has been realized.

We consider the dynamics of the one-dimensional nearestneighbor Ising model without a transverse field or decoherence. This is described by the Hamiltonian

$$
H_{\mathrm{I}}=-J \sum_{i} \sigma_{i}^{z} \sigma_{i+1}^{z},
$$

where $J$ is the interaction term. We study the dynamics of this model initiated from the product state $|\theta \theta \theta \cdots\rangle$, where

$$
|\theta\rangle=\cos \theta / 2|\uparrow\rangle+\sin \theta / 2|\downarrow\rangle .
$$

With no loss of generality, we have assumed that the spins are initially in the $x-z$ plane. One experimentally straightforward way to produce this initial state is to rotate each spin, after it is prepared in the single-spin ground state $|\downarrow\rangle$, by using a global laser pulse. This is the standard first step of any Ramsey experiment $[26,77]$. The system is then allowed to evolve from the initial state under $H_{\mathrm{I}}$.

Over time, correlations build up in the system. References $[76,78,79]$ calculate the single-spin expectations and two-spin correlations. The single-spin observables are

$$
\begin{gathered}
b_{k}^{z}(t)=\left\langle\sigma_{k}^{z}(t)\right\rangle=\cos \theta, \\
b_{k}^{x}(t)=\left\langle\sigma_{k}^{x}(t)\right\rangle=\operatorname{Re}\left(\sin \theta\left[g^{+}(J t)\right]^{2}\right), \\
b_{k}^{y}(t)=\left\langle\sigma_{k}^{y}(t)\right\rangle=\operatorname{Im}\left(\sin \theta\left[g^{+}(J t)\right]^{2}\right),
\end{gathered}
$$

where

$$
g^{ \pm}(x)=\cos ^{2}(\theta / 2) e^{-i 2 x} \pm \sin ^{2}(\theta / 2) e^{i 2 x} .
$$

The two-point correlations are

$$
\begin{aligned}
& c_{j k}^{x x}(t)= \frac{1}{4}\left(c_{j k}^{++}+c_{j k}^{--}+c_{j k}^{+-}+c_{j k}^{-+}\right), \\
& c_{j k}^{y y}(t)= \frac{1}{4}\left(c_{j k}^{+-}+c_{j k}^{-+}-c_{j k}^{++}-c_{j k}^{--}\right), \\
& c_{j, k}^{z z}(t)=b_{j}^{z}(t) b_{k}^{z}(t), \\
& c_{j k}^{x y}(t)= \frac{1}{4 i}\left(c_{j k}^{++}-c_{j k}^{--}-c_{j k}^{+-}+C_{j k}^{-+}\right), \\
& c_{j k}^{x z}(t)=\frac{1}{2}\left(c_{j k}^{+z}+c_{j k}^{-z}\right), \\
& c_{j k}^{y z}(t)=\frac{1}{2 i}\left(c_{j k}^{+z}-c_{j k}^{-z}\right),
\end{aligned}
$$

where

$$
\begin{aligned}
& c_{k, k+n}^{+ \pm}(t)= \begin{cases}\sin ^{2} \theta\left[g^{+}(J t) g^{+}( \pm J t)\right] & \text { for } n=1 \\
\sin ^{2} \theta g^{+}(J t \pm J t) & \\
\times\left[g^{+}(J t) g^{+}( \pm J t)\right] & \text { for } n=2 \\
\sin ^{2} \theta\left[g^{+}(J t) g^{+}( \pm J t)\right]^{2} & \text { for } n>2,\end{cases} \\
& c_{k, k+n}^{+z}(t)= \begin{cases}\sin \theta g^{-}(J t) g^{+}(J t) & \text { for } n=1 \\
\sin \theta\left[g^{+}(J t)\right]^{2} & \text { for } n>1,\end{cases}
\end{aligned}
$$

and the other correlations can be calculated from the identities

$$
\begin{aligned}
& c_{k, k+n}^{- \pm}(t)=\left[c_{k, k+n}^{+\mp}(t)\right]^{*}, \\
& c_{k, k+n}^{-z}(t)=\left[c_{k, k+n}^{+z}(t)\right]^{*} .
\end{aligned}
$$

Figure 4(a) shows the coherent dynamics for the initial state $\theta=\pi / 2$. The NN correlations are described by a clover oriented with its petals pointing at $45^{\circ}$ in the $y z$ plane. It grows and shrinks periodically. At its maximum size, the clover fattens to have some wheel-and-axle character.

Figure 4(b) shows the same dynamics for the initial state $\theta=\pi / 4$. (This is the same example as that used in Fig. 1.) The dynamics is qualitatively similar to the $\pi / 2$ case, except (i) the initial CMV is rotated to orient the clover perpendicular to the initial Bloch vector, (ii) the clover is slightly smaller [note that the size of the CMVs has been magnified for easier visualization in Fig. 4(b)], and (iii) a precession about the $z$ axis is superimposed. This precession is a mean-field effect in which every spin experiences a local field along the $z$ axis due to the interactions with the background spins. Although the CMV precession closely follows the Bloch vectors, there are small differences between the two.

As expected for a model with short-range interactions, next-nearest-neighbor (NNN) correlations are smaller than the NN correlations. More interestingly, the structure of the NNN CMV is qualitatively different. It is a simple dumbbell that grows and shrinks. In the Supplemental Material, we have movies for the coherent dynamics for longer times [80].

This dynamics is exemplary of the utility of the CMVs. Remarkably, although the components of the correlations evolve in an extremely complicated manner, as shown in 


\section{tJ}

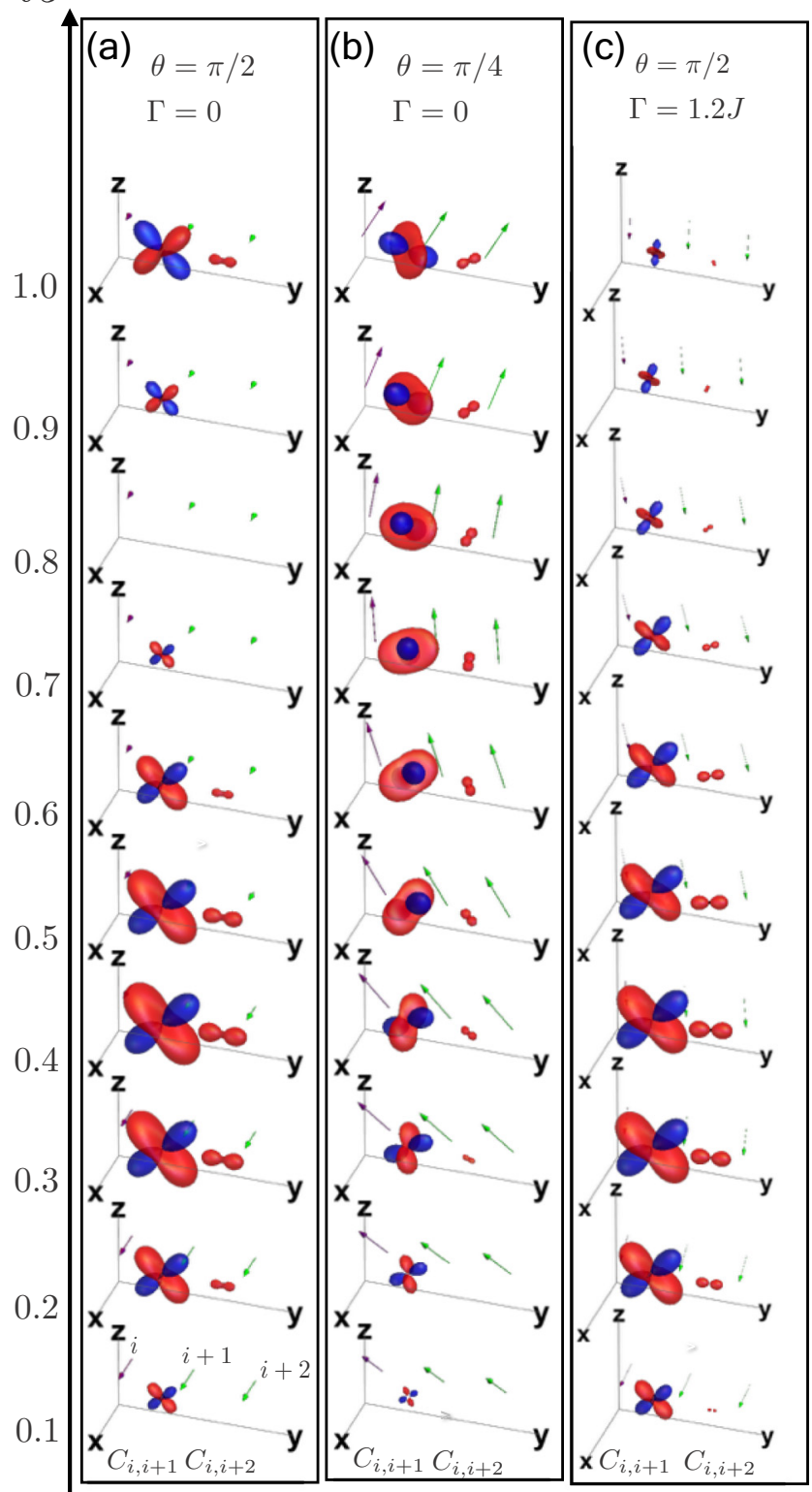

FIG. 4. Dynamics of the nearest-neighbor one-dimensional Ising model (no transverse field) initiated from a state with all the spins aligned. Arrows (green and purple) indicate Bloch vectors of three adjacent spins belonging to an infinite spin chain. At every instant, the correlations are depicted with respect to the first spin (purple). (a) Coherent Ising dynamics (no dissipation) for $\theta=\pi / 2$ initial state. (b) Ising dynamics for $\theta=\pi / 4$ initial state. (c) Ising dynamics with dissipation for the same $\theta$ as (a). The CMVs for (b) and (c) have been magnified by factors of 1.2 and 1.5 , respectively, to be comparable to (a) for convenient visualization.

Figs. 1(b) and 1(c), the CMVs behave simply: a growing and shrinking clover superimposed on a rotation. If one were to consider the dynamics of the many components $C_{i j}^{\alpha \beta}$, it would be difficult to describe them, remember them, or form a mental picture of them. In contrast, the CMVs provide a description of the dynamics in terms of growth and rotation of figures that is simple to describe, remember, and visualize.

\section{B. Ising model with decoherence}

There are deep questions about the fate of correlations in open quantum systems that the CMVs may be useful in illuminating. Furthermore, any system is inevitably coupled, however weakly, to some environment, so understanding the effects of this decoherence on the correlations is an important practical goal.

With this in mind, we consider the dynamics of the Ising model in the presence of an incoherent spontaneous emission. We choose this form of decoherence both due to its simplicity and because it is an important decoherence mechanism in many experiments. The system's reduced density matrix satisfies a master equation with Markovian dissipation,

$$
\dot{\rho}=-i\left[H_{\mathrm{I}}, \rho\right]+\mathcal{L}(\rho),
$$

where $\mathcal{L}(\rho)$ is the Lindblad term,

$$
\mathcal{L}(\rho)=\frac{\Gamma}{2} \sum_{j}\left(2 \sigma_{j}^{-} \rho \sigma_{j}^{+}-\sigma_{j}^{+} \sigma_{j}^{-} \rho-\rho \sigma_{j}^{+} \sigma_{j}^{-}\right) .
$$

From Refs. [81,82], the single-spin expectations are

$$
\begin{gathered}
b_{k}^{z}(t)=\left\langle\sigma_{k}^{z}(t)\right\rangle=\left(e^{-\Gamma t}-1\right)+e^{-\Gamma t} \cos \theta, \\
b_{k}^{x}(t)=\left\langle\sigma_{k}^{x}(t)\right\rangle=\operatorname{Re}\left[e^{-\Gamma t} \Phi^{2}(J, t)\right], \\
b_{k}^{y}(t)=\left\langle\sigma_{k}^{y}(t)\right\rangle=\operatorname{Im}\left[e^{-\Gamma t} \Phi^{2}(J, t)\right],
\end{gathered}
$$

where

$$
\Phi(J, t)=e^{-\Gamma t / 2}\left[\cos (s t)+\frac{\Gamma t}{2} \operatorname{sinc}(s t)\right],
$$

with $\operatorname{sinc}(x)=\sin (x) / x$ and

$$
s=2\left(i \frac{\Gamma}{4}-J\right) \text {. }
$$

The correlations $c_{j k}^{\alpha \beta}$ can be calculated using Eqs. (16)-(21) and Eqs. (24) and (25), where, for the dynamics with decoherence,

$$
\begin{gathered}
c_{k, k+n}^{+ \pm}(t)= \begin{cases}e^{-2 \Gamma t} \Phi(J, t) \Phi( \pm J, t) & \text { for } n=1 \\
e^{-2 \Gamma t} \Phi(J \pm J, t) \Phi(J, t) \Phi( \pm J, t) & \text { for } n=2 \\
e^{-2 \Gamma t}[\Phi(J, t) \Phi( \pm J, t)]^{2} & \text { for } n>2,\end{cases} \\
c_{k, k+n}^{+z}(t)= \begin{cases}e^{-\Gamma t} \Psi(J, t) \Phi(J, t) & \text { for } n=1 \\
e^{-\Gamma t} \Phi^{2}(J, t) & \text { for } n>1\end{cases}
\end{gathered}
$$

where

$$
\Psi(J, t)=e^{-\Gamma t / 2}\left(i s-\frac{\Gamma}{2}\right) t \operatorname{sinc}(s t) .
$$

Figure 4(c) depicts the CMV for the Ising dynamics with decoherence for $\theta=\pi / 2$ (also see the movie in the Supplemental Material [80]). The shape for the NN correlations is a clover throughout the dynamics.

Despite the similarities, adding decoherence qualitatively modifies the $\theta=\pi / 2$ CMV dynamics in two ways. The first, trivial effect is that eventually the CMV vanishes. This happens because the correlations vanish when all of the spins have damped to the ground state (pointing along the negative $z$ axis). 
Second, the CMV precesses (rotates about the $z$ axis), starting around $t J \sim 0.7$. This is more visually apparent in the movie given in the Supplemental Material [80]. The precession occurs only at later times because, unlike the coherent $\theta=\pi / 4$ dynamics, the rate of precession of the CMV (and Bloch vectors) with decoherence grows with time. In fact, it is proportional to the $z$ component of the Bloch vector, as this is essentially a mean-field effect similar to that occurring for $\theta=\pi / 4$.

\section{Fermi-Hubbard model}

In this section, we study the dynamics of correlations of the Fermi-Hubbard model after a sudden quench to the noninteracting limit, which is similar to various quenches considered in Refs. $[24,25,83,84]$. This can be accomplished by sweeping across a Feshbach resonance or sufficiently lowering the lattice depth. The equilibrium properties of the FermiHubbard model are well studied in cold atoms, and studying its quench dynamics is an exciting frontier [85-93]. Great progress has been made in terms of imaging fermions [94-96], achieving Mott states [97-99], and generating short-range antiferromagnetic (AFM) correlations [86,89,99-103]. With these techniques, experimentally preparing the states that we study and measuring the resulting correlations has become feasible.

As in prior sections, we concentrate on the one-dimensional case for simplicity. The Fermi-Hubbard Hamiltonian is

$$
H=-\mathcal{J} \sum_{\langle i, j\rangle, \sigma} c_{i \sigma}^{\dagger} c_{j \sigma}+U \sum_{i} n_{i \uparrow} n_{i \downarrow},
$$

where $\mathcal{J}$ is tunneling, $U$ is the on-site interaction, and $\sigma=\uparrow$, $\downarrow$. The $c_{i \sigma}^{\dagger}\left(c_{i \sigma}\right)$ are fermionic creation (annihilation) operators and $n_{i \sigma}=c_{i \sigma}^{\dagger} c_{i \sigma}$ is the number operator for spin $\sigma$ at site $i$. We calculate the correlations for $U=0$ between sites $q$ and $r$,

$$
\begin{aligned}
\left\langle\sigma_{q}^{\mu}(t) \sigma_{r}^{\nu}(t)\right\rangle= & \sum_{a b c d} \sigma_{a b}^{\mu} \sigma_{c d}^{\nu}\left\langle c_{q a}^{\dagger}(t) c_{q b}(t) c_{r c}^{\dagger}(t) c_{r d}(t)\right\rangle \\
= & \sum_{i j k l} A_{q i}^{*}(t) A_{q j}(t) A_{r k}^{*}(t) A_{r l}(t) \\
& \times \sum_{a b c d} \sigma_{a b}^{\mu} \sigma_{c d}^{\nu}\left\langle c_{i a}^{\dagger}(0) c_{j b}(0) c_{k c}^{\dagger}(0) c_{l d}(0)\right\rangle,
\end{aligned}
$$

where $a, b, c, d \in\{\uparrow, \downarrow\}$. The $A_{j l}$ are the propagators,

$$
\begin{aligned}
A_{j l} & =\frac{1}{N} \sum_{k} \exp \left[i k(j-l)+i E_{k} t\right] \\
& =(-i)^{|j-l|} J_{|j-l|}(2 \mathcal{J} t) .
\end{aligned}
$$

Here, $J_{m}(z)$ is a Bessel function of the first kind and

$$
E_{k}=-2 \mathcal{J} \cos (k)
$$

is the dispersion relation where we have assumed unit lattice spacing.

We assume our initial state to be a product state, and as a result the expectation value of a many-body operator can be factored into a product of single-site expectation values. For an arbitrary product state, we find that the Bloch vectors are

$$
b_{i}^{\mu}(t)=\sum_{a b} \sigma_{a b}^{\mu} \sum_{j}\left|A_{i j}(t)\right|^{2} f_{j}(a b)
$$

and the correlation functions are

$$
\begin{aligned}
c_{q r}^{\mu \nu}(t)= & \sum_{a b c d} \sigma_{a b}^{\mu} \sigma_{c d}^{\nu}\left(\sum_{i}\left|A_{q i}(t)\right|^{2}\left|A_{r i}(t)\right|^{2}\right. \\
& \times\left\{g_{i}(a b c d)-f_{i}(a b) f_{i}(c d)-f_{i}(a d)\left[\delta_{b c}-f_{i}(c b)\right]\right\} \\
& +\left[\sum_{i}\left|A_{q i}(t)\right|^{2} f_{i}(a b)\right]\left[\sum_{k}\left|A_{r k}(t)\right|^{2} f_{k}(c d)\right] \\
& +\left[\sum_{i} A_{q i}^{*}(t) A_{r i}(t) f_{i}(a c)\right] \\
& \left.\times\left\{\sum_{j} A_{q j}(t) A_{r j}^{*}(t)\left[\delta_{b c}-f_{j}(c b)\right]\right\}\right)
\end{aligned}
$$

where we have defined

$$
\begin{gathered}
f_{j}(a b)=\left\langle c_{j a}^{\dagger} c_{j b}\right\rangle, \\
g_{j}(a b c d)=\left\langle c_{j a}^{\dagger} c_{j b} c_{j c}^{\dagger} c_{j d}\right\rangle,
\end{gathered}
$$

with the expectation values taken at time $t=0$.

Although our calculations allow general product states as initial conditions, here we concentrate on a specific initial state, which we refer to as the canted antiferromagnetic product state. This initial state can be viewed as an antiferromagnetic state $\langle\uparrow \downarrow \cdots\rangle$ that is then canted by adding an $x$ component to each spin. Similar initial states can be prepared experimentally using magnetic field gradients [104], polarization field gradients in optical superlattices $[105,106]$, or spin-changing collisions in double wells [107-110]. As an example, we focus on a product state,

$$
|\psi\rangle=\bigotimes_{k} \begin{cases}|\nearrow\rangle_{k}, & k \text { even } \\ |\searrow\rangle_{k}, & k \text { odd }\end{cases}
$$

where $\quad|\nearrow\rangle=\cos (\pi / 8)|\downarrow\rangle+\sin (\pi / 8)|\uparrow\rangle \quad$ and $\quad|\searrow\rangle=$ $\cos (3 \pi / 8)|\downarrow\rangle+\sin (3 \pi / 8)|\uparrow\rangle \quad$ [refer to Eq. (11)]. The qualitative features in the dynamics are mostly independent of the specific choice of the initial state. This state is chosen to give concrete examples of CMVs as shown in Fig. 5 that highlight symmetries that are not obviously connected to the symmetry or orientation of the Bloch vectors or that of the Hamiltonian. For this initial state, we have

$$
\begin{gathered}
f_{j}(a b)=\left[\frac{1}{2}+\frac{(-1)^{j+\delta_{a \downarrow}}}{2 \sqrt{2}}\right] \delta_{a b}+\frac{1-\delta_{a b}}{2 \sqrt{2}}, \\
g_{j}(a b c d)=\left[\frac{1}{2}+\frac{(-1)^{j+\delta_{a \downarrow}}}{2 \sqrt{2}}\right] \delta_{b c} \delta_{a d}+\frac{\delta_{b c}\left(1-\delta_{a d}\right)}{2 \sqrt{2}} .
\end{gathered}
$$

Figure 5 depicts the dynamics of a system that starts with a canted antiferromagnet as its initial state and then is quenched to a noninteracting system (also see the movie in the Supplemental Material [80]). We find that the maximum correlation with respect to the first spin (purple) initially shifts over time from NN to NNN. At longer times, the influence of correlations 


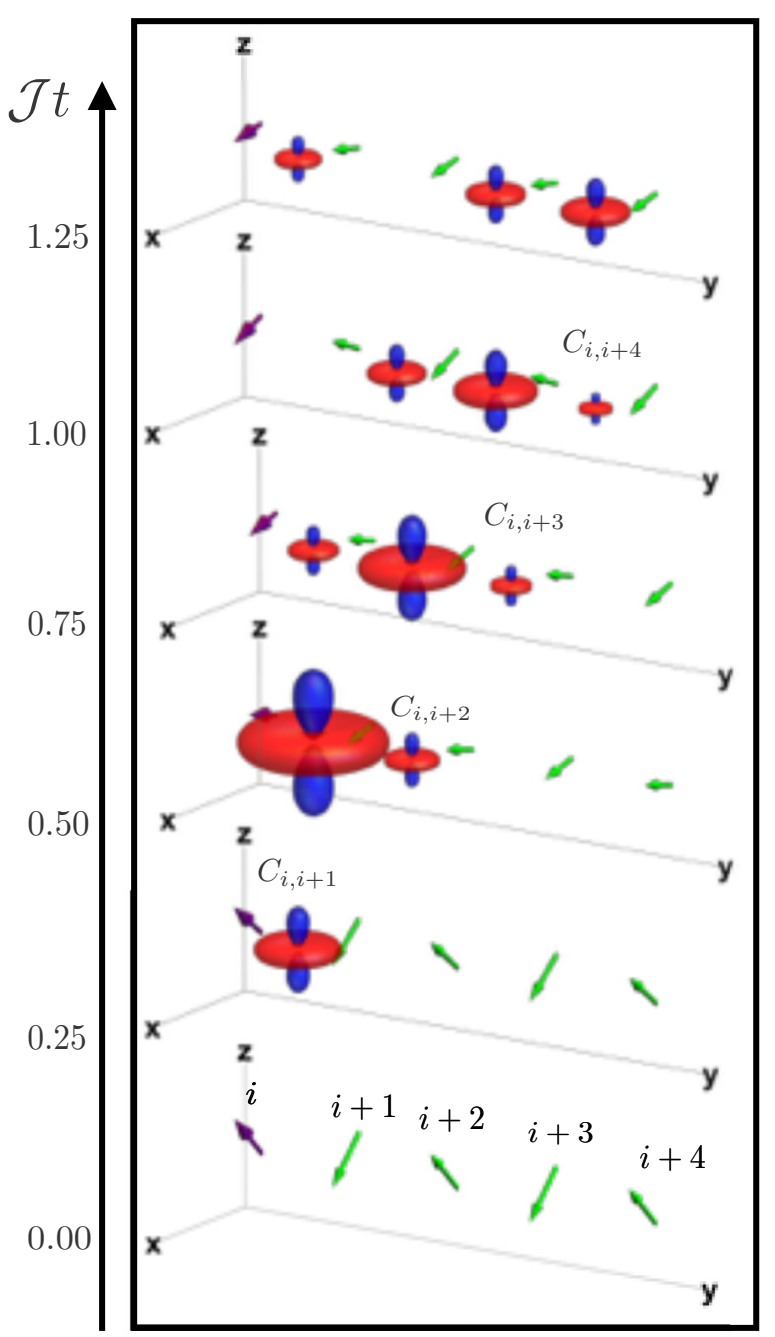

FIG. 5. Dynamics of noninteracting spin-1/2 fermions initiated from an initial one-spin-per-site (Mott-insulating) staggered spin product state is shown. Time advances vertically, from bottom to top, with each panel corresponding to the evolution of the correlation at a given time.

continues to spread over wider spatial regions. The propagation of correlations in a lattice is commonly referred to as the light cone [111-115]. It should also be noted that the maximum size any CMV can attain decreases with distance and time. The CMVs have the wheel-and-axle shape with a U(1) symmetry around the $z$ axis. This is particularly interesting because it contrasts with both the initial state's $Z_{2}$ glide symmetry and the Hamiltonian's SU(2) symmetry. The geometric object representing the correlations uncovers emergent symmetries that are not trivially related to either the Hamiltonian or the initial state. This surprising observation reveals features in nonequilibrium dynamics that remain to be understood.

\section{Transverse Ising phase diagram}

In contrast to the previous sections, which involved nonequilibrium dynamics, here we focus on equilibrium correlations. Specifically, we study the one-dimensional transverse
Ising model with $\mathrm{NN}$ interactions,

$$
H_{\mathrm{T}}=H_{\mathrm{I}}-h \sum_{i} \sigma_{i}^{x},
$$

where $h>0$ is the transverse field. We define $g=h / J$. With this definition, $g_{c}=1$ is the critical value of $g$ that separates the ferromagnetic and paramagnetic phases at zero temperature [13]. The single-spin expectations are [116]

$$
\begin{gathered}
\left\langle\sigma^{x}\right\rangle=D_{0}, \\
\left\langle\sigma^{y}\right\rangle=0, \\
\left\langle\sigma^{z}\right\rangle=0,
\end{gathered}
$$

and the correlations are [116]

$$
\begin{gathered}
C_{i, i+n}^{x x}=\left|\begin{array}{cc}
D_{0} & D_{n} \\
D_{-n} & D_{0}
\end{array}\right|-D_{0}^{2} \\
=-D_{-n} D_{n}, \\
C_{i, i+n}^{y y}=\left|\begin{array}{cccc}
D_{-1} & D_{0} & \ldots & D_{n-2} \\
D_{-2} & D_{-1} & \ldots & D_{n-3} \\
\vdots & \vdots & \ddots & \vdots \\
D_{-n} & D_{-n+1} & \ldots & D_{-1}
\end{array}\right|, \\
C_{i, i+n}^{z z}=\left|\begin{array}{cccc}
D_{1} & D_{2} & \ldots & D_{n} \\
D_{0} & D_{1} & \ldots & D_{n-1} \\
\vdots & \vdots & \ddots & \vdots \\
D_{-n+2} & D_{-n+3} & \ldots & D_{1}
\end{array}\right|, \\
C_{i, j}^{x y}=C_{i, j}^{y z}=C_{i, j}^{x z}=0,
\end{gathered}
$$

where

$$
D_{n}=-\int_{-\pi}^{\pi} \frac{d k}{2 \pi}\left[1-2 v_{k}^{2}+2 i u_{k} v_{k}\right] e^{i k n} \tanh \left(\frac{\omega_{k}}{2 T}\right) .
$$

Here, $T$ is the temperature, we have set the Boltzmann constant $k_{B}=1, \omega_{k}$ is given as

$$
\omega_{k}=2 J \sqrt{1+g^{2}-2 g \cos k},
$$

and $u_{k}$ and $v_{k}$ are given as

$$
\begin{aligned}
& u_{k}=\frac{2 J \sin k}{\sqrt{2 \omega_{k}\left[\omega_{k}-2 J(g-\cos k)\right]}}, \\
& v_{k}=\frac{\omega_{k}-2 J(g-\cos k)}{\sqrt{2 \omega_{k}\left[\omega_{k}-2 J(g-\cos k)\right]}} .
\end{aligned}
$$

Figure 6 shows CMVs in different regions of the equilibrium phase diagram of the transverse Ising model. At $g=0$, we have a dumbbell-shaped CMV for all values of $T$. The dumbbell shape and its evolution with $g$ and $T$ is of some physical interest. To obtain some insight into this, note that in the (purely classical) limit of a Hamiltonian depending only on the $\sigma_{i}^{z}$, the density matrix is $\sum_{q r \in\{\uparrow \downarrow\}} \rho_{q r}|q r\rangle\langle q r|$. This has correlations along $z$ and vanishing $x$ and $y$ correlations, leading to a 


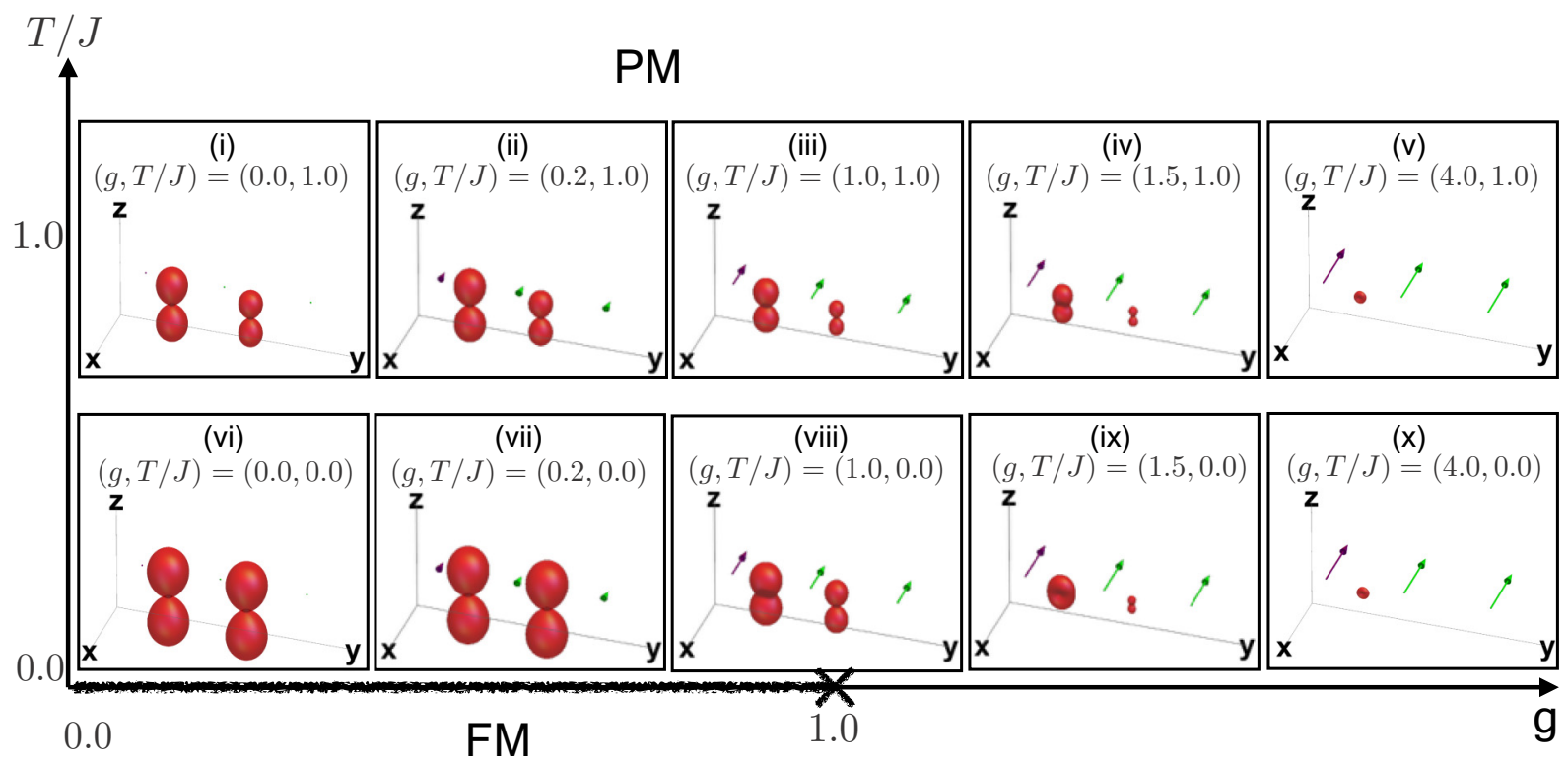

FIG. 6. Correlations are shown throughout the equilibrium phase diagram for a one-dimensional NN transverse-field Ising model, where FM stands for ferromagnetic phase and PM for the paramagnetic phase. The FM phase strictly exists only at $T=0$. Each panel in (i) $-(\mathrm{vi})$ contains the CMVs corresponding to a specific value for the parameters $(g, T / J)$. Left and right CMVs in each panel are NN and NNN correlations, respectively. The Bloch vectors have small but finite lengths at small values of $g$.

dumbbell shape. Upon perturbing this system with a transverse field, quantum fluctuations create coherences and correlations involving the $x$ and $y$ directions. This is reminiscent of the fact that in the similar steering ellipsoid visualization of Ref. [56], a single nonzero eigenvalue is insufficient to obtain entanglement.

On increasing the transverse field $g$, there are two significant trends. First the Bloch vectors grow along the $x$ direction as expected. Second, the CMVs gradually change from a dumbbell to a disklike shape [see Fig. 6(vi)], which indicates that there are nonzero correlations in the $y z$ plane. This is due to quantum fluctuations.

For extremely large $g$, a new symmetry emerges corresponding to rotations about the $x$ axis. One way to understand this is by the mapping presented in Ref. [114] showing that this model for $g \rightarrow \infty$ maps to an XX model with this symmetry. However, we note that by examining the correlations with CMVs, the emergent symmetry is immediately apparent even without prior knowledge of this mapping.

Increasing temperature leads to a dumbbell shape in all cases. This is clearest in Fig. 6(iii). This occurs as the thermal fluctuations overwhelm the quantum fluctuations, even though the correlations remain strong.

The NNN correlations are smaller in magnitude than the $\mathrm{NN}$ correlations for all values of $g$. However, this is visually apparent only away from the $(g, T)=0$ point.

\section{CONCLUSION}

We generate a three-dimensional geometric object by associating spin-correlation matrices with a quadratic form. We apply this visualization scheme to various prototypical correlated quantum states, and to a variety of models relevant for ultracold-atom experiments. We demonstrate that phenomena that look complicated and mysterious when analyzed by the components of their correlations become simple and intuitive when described geometrically. For example, the CMVs describing correlations often pulsate and rotate in fairly simple ways.

The simplicity uncovered by our work opens up exciting questions: Are there ways to determine qualitatively the shapes that appear in the CMVs, especially for models that are not exactly solvable? Are there general rules that underly the simple motions of the CMVs? For example, can we understand under what circumstances CMVs grow and shrink, or when they rotate in a simple manner, for example at a steady rate around a fixed axis?

It will also be interesting to uncover how a given manipulation of the CMV shape can be engineered via Hamiltonian evolution or other quantum operations. Use of this insight to control the state would have applications in quantum information and many-body physics. It would be intriguing to combine this with the methods in Ref. [117], where each edge on their graph would be associated with a CMV between the corresponding pair of spins.

Two-spin CMVs can be generalized in various ways. One can associate three- and higher-spin correlations with geometric objects (see Appendix B). The number of correlation components grows exponentially with the number of spins involved, $N_{s}$; specifically, there are $3^{N_{s}}$ components. Thus the correlations may have a complexity far exceeding two-spin correlations. It would be especially interesting if equally simple motions underlie these higher-spin correlations.

\section{ACKNOWLEDGMENTS}

K.R.A.H. acknowledges the Aspen Center for Physics for its hospitality while part of this work was performed. J.H. acknowledges the SCI REU program for giving him the opportunity to do this work at Rice University. This work was 
supported with funds from the Welch Foundation, Grant No. C-1872. We also acknowledge discussions with Charles Xu and Kenneth Wang.

\section{APPENDIX A: VISUALIZING ASYMMETRIC CORRELATION MATRIX}

Our method of using quadratic forms depends only on the symmetric part of the correlation matrix. To visualize an arbitrary correlation matrix $C_{i j}$, note that it can be split into a sum of symmetric and antisymmetric parts, $\mathcal{S}_{i j}^{\alpha \beta}=(1 / 2)\left(C_{i j}^{\alpha \beta}+C_{i j}^{\beta \alpha}\right)$ and $\mathcal{A}_{i j}^{\alpha \beta}=(1 / 2)\left(C_{i j}^{\alpha \beta}-C_{i j}^{\beta \alpha}\right)$, respectively. The former may be visualized as in the main text, while the antisymmetric piece can be identified as usual with a pseudovector $(a, b, c)$. The pseudovector can be visualized as an arrow (similar to the Bloch vectors) or by level sets of linear forms $L(\vec{r})=$ $a x+b y+c z$ (perhaps adding an appropriate denominator to render them compact), much as we use level sets of $Q(\vec{r})$ for the symmetric piece. Another method to handle asymmetric correlations is to take singular-value decompositions of the relevant matrix and consider left and right eigenvectors [55].

\section{APPENDIX B: GENERALIZATION TO MANY-SPIN CORRELATIONS}

Let the connected correlation matrix for $N$ spin-1/2's be the rank- $N$ tensor $C_{i j \ldots N}^{\mu \nu \ldots \gamma}=\left\langle\sigma_{i}^{\mu} \sigma_{j}^{\nu} \cdots \sigma_{N}^{\gamma}\right\rangle-\cdots$, where “..." indicates terms to be subtracted in order to produce the desired connected correlation. One can define a CMV by

$$
F\left(C_{i j \ldots N}, \mathbf{r}\right)=\sum_{\mu, \nu \ldots \gamma \in\{x, y, z\}} C_{i j \ldots N}^{\mu \nu \ldots \gamma} r^{\mu} r^{\nu} \ldots r^{\gamma} .
$$

Similar to the two-spin case, the extent of the geometrical object in a direction $\alpha$ measures the size of the correlations in that direction. Also similar to the two-spin case, this is now sensitive only to the totally permutationally symmetric component of the correlations.

\section{APPENDIX C: IRREDUCIBLE COMPONENTS OF $N$-BODY CORRELATIONS}

In order to gain insight into the correlations, it can be useful to decompose the tensor $C_{i j \ldots N}$ into its spherically irreducible components, a method employed in Ref. [56]. This method makes more explicit the properties of the figures under global spin rotations and includes both symmetric and asymmetric correlations in a uniform manner. We decompose

$$
C_{i j \ldots N}=\sum_{l \in L} \sum_{m=-l}^{l} a_{l m}\left(C_{i j \ldots N}\right) T_{l m},
$$

where $L=\{0,1, \ldots, N\}, T_{l m}$ are the components of the spherical tensor $T_{l}$ with rank $l$, and $a_{l m}$ are the coefficients of the expansion, which characterize $C_{i j \ldots N}$. To visualize $C_{i j \ldots N}$, one can plot

$$
f\left(C_{i j \ldots N} ; \theta, \phi\right)=\sum_{l \in L} \sum_{m=-l}^{l} a_{l m}\left(C_{i j \ldots N}\right) Y_{l m}(\theta, \phi),
$$

where $Y_{l m}(\theta, \phi)$ are the spherical harmonics. If one prefers a three-dimensional image, similar to the CMVs, one can add a dependence on $r$ and plot level sets; e.g., one can plot level sets of $f\left(C_{i j \ldots N} ; \theta, \phi\right) /\left(1+r^{2}\right)^{3 / 2}$.

A convenient extension to this is to consider associating each irreducible sector with its own geometric object. Thus, one associates the $l=0$ terms with one object, the $l=1$ terms with another, and so on. To do this, one restricts the sum in Eq. (C2) to the $l$ corresponding to the object. This representation is convenient because under global spin rotations, objects of shapes in the rank- $l$ irreducible space transform only among each other, and not into shapes in other spaces.

The case of two spin- $1 / 2$ 's provides a familiar example. The correlation tensor can be decomposed into a sum of $l=0,1,2$ components $C=C_{0}+C_{1}+C_{2}$ (suppressing $i j \ldots N$ indices for brevity), with

$$
\begin{gathered}
C_{0}^{\mu \nu}=\delta_{\mu \nu} \frac{\operatorname{Tr}(C)}{3}, \\
C_{1}^{\mu \nu}=\frac{C^{\mu \nu}-C^{\nu \mu}}{2}, \\
C_{2}^{\mu \nu}=\frac{C^{\mu \nu}+C^{\nu \mu}}{2}-C_{0} .
\end{gathered}
$$

To compare this decomposition with the method used in the main text, note that there we plot the entire symmetric part of $C$, i.e., $C_{2}^{\mu \nu}+C_{0}^{\mu \nu}$. This more directly allows one to read off the size of the total correlations in a given direction, but at the cost of a slightly more complicated transformation under rotations.

\section{APPENDIX D: RELATING THE SIZE OF CMV TO THE MAGNITUDE OF CORRELATIONS}

In the main text, Sec. III discussed how to read information off of a CMV, and in particular it claimed that the extent of the CMV along a unit direction $\hat{\mathbf{e}}$ indicates the size of "connected correlations in that direction," $\mathcal{C}_{i j}(\hat{\mathbf{e}})=$ $\left\langle\left(\sigma_{i} \cdot \hat{\mathbf{e}}\right)\left(\sigma_{j} \cdot \hat{\mathbf{e}}\right)\right\rangle-\left\langle\sigma_{i} \cdot \hat{\mathbf{e}}\right\rangle\left\langle\sigma_{j} \cdot \hat{\mathbf{e}}\right\rangle$. The main step towards this connection is Eq. (8), which established that $\mathcal{C}_{i j}(\hat{\mathbf{e}})$ is equal to $Q\left(C_{i j}, \hat{\mathbf{e}}\right)$. Then, the interpretation of the CMV is validated by noting that the value of $Q\left(C_{i j}, \hat{\mathbf{e}}\right)$ determines the extent of the CMV along ê.

The purpose of this appendix is to establish this intuitive link between $Q\left(C_{i j}, \hat{\mathbf{e}}\right)$ and the size of the CMV along $\hat{\mathbf{e}}$, and to give more insight into it. Figure 7 demonstrates the key idea: as $Q\left(C_{i j}, \hat{\mathbf{e}}\right)$ is increased, the graph of $Q_{f}$ as a function of the distance along that direction is increased. Due to the peaked shape of this function, the inner and outer parts of the level set get farther apart, increasing the size of the shape in that direction.

To establish this geometric fact algebraically, we will calculate the separation of the inner and outer level sets where $Q\left(C_{i j}, r^{*}\right)=P$, for $r_{\text {in }}^{*}$ and $r_{\text {out }}^{*}$, respectively. We will assume that the inner level set $r_{\text {in }}^{*} \ll 1$ and $r_{\text {out }}^{*} \gg 1$, not because we expect this to be valid generally, but because this case is illustrative of the connection that we seek to establish and the math is particularly simple. Equation (5) can be 


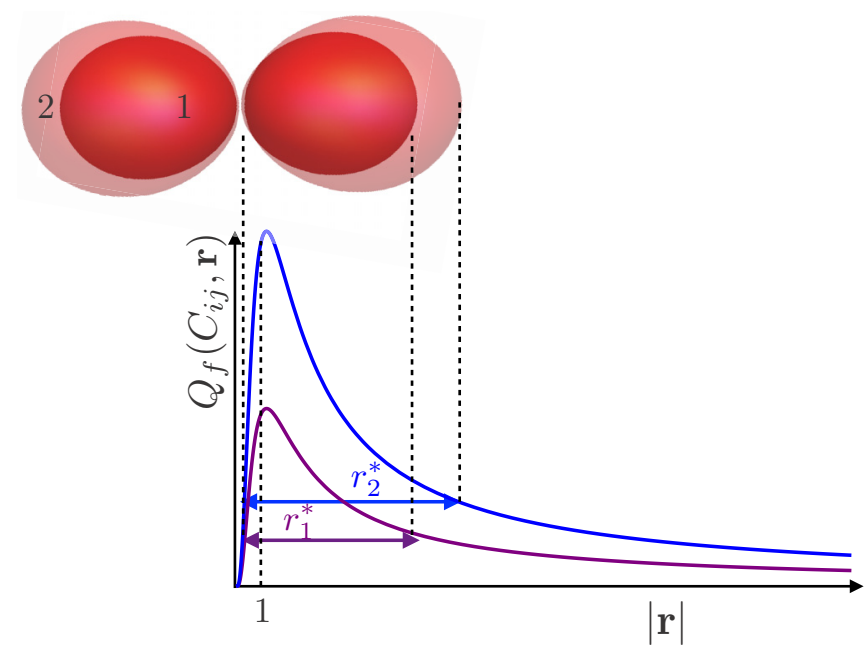

FIG. 7. Two CMVs are shown corresponding to level sets of $Q_{f}\left(C_{i j}=0.1, r\right)=0.1$ (labeled as 1) and $Q_{f}\left(C_{i j}=0.2, r\right)=0.1$ (labeled as 2). The size of each lobe belonging to CMV 1,2 is given by $r_{1,2}^{*}$, as suggested by Eq. (D2).

rewritten as

$$
\begin{aligned}
Q_{f} & =\frac{\left\langle\left(\sigma_{i} \cdot \mathbf{r}\right)\left(\sigma_{j} \cdot \mathbf{r}\right)\right\rangle-\left\langle\sigma_{i} \cdot \mathbf{r}\right\rangle\left\langle\sigma_{j} \cdot \mathbf{r}\right\rangle}{\left(1+r^{2}\right)^{3 / 2}} \\
& =\frac{r^{2}\left[\left\langle\left(\sigma_{i} \cdot \hat{r}\right)\left(\sigma_{j} \cdot \hat{r}\right)\right\rangle-\left\langle\sigma_{i} \cdot \hat{r}\right\rangle\left\langle\sigma_{j} \cdot \hat{r}\right\rangle\right]}{\left(1+r^{2}\right)^{3 / 2}} \\
& =\frac{r^{2}}{\left(1+r^{2}\right)^{3 / 2}} \mathcal{C}_{i j}(\hat{\mathbf{e}}) .
\end{aligned}
$$

In the limit considered, $r_{\text {out }}^{*}=\mathcal{C}_{i j}(\hat{\mathbf{e}}) / P$ and $r_{\text {in }}^{*}=\sqrt{P / \mathcal{C}_{i j}(\hat{\mathbf{e}})}$. Thus the size of a CMV along the direction $\mathbf{r}$ is given as

$$
r_{\text {size }}^{*}=r_{\text {out }}^{*}-r_{\text {in }}^{*}=\left[\mathcal{C}_{i j}(\hat{\mathbf{e}}) / P-\sqrt{P / \mathcal{C}_{i j}(\hat{\mathbf{e}})}\right]
$$

From the above expression, one sees that as the magnitude of $\mathcal{C}_{i j}(\hat{\mathbf{e}})$ increases, so does the size of the CMV along that direction. This interpretation still holds without making the asymptotic assumptions we have here, but the CMV size along $\hat{\mathbf{e}}$ is no longer related to the value of $Q\left(C_{i j}, \hat{\mathbf{e}}\right)$ by a simple proportionality.

\section{APPENDIX E: COMPARING CMV WITH ANALOGOUS METHODS OF VISUALIZATION}

The crucial ideas in our paper and its conclusion-that geometrically visualizing two-body correlations unveils simple features in many-body physics - is not tied to the particular CMV visualization method that we have chosen. Alternative visualizations of correlations, for example based on Wigner functions [53-56] or eigenvectors, can reveal the same physics, but they differ in their detailed interpretation, aesthetics, and ease of implementation. The objective of this appendix is to compare these methods with the CMVs. Figure 8 summarizes this comparison.

We consider two alternatives to CMVs that encode the same information - the two-spin correlations-in visually different

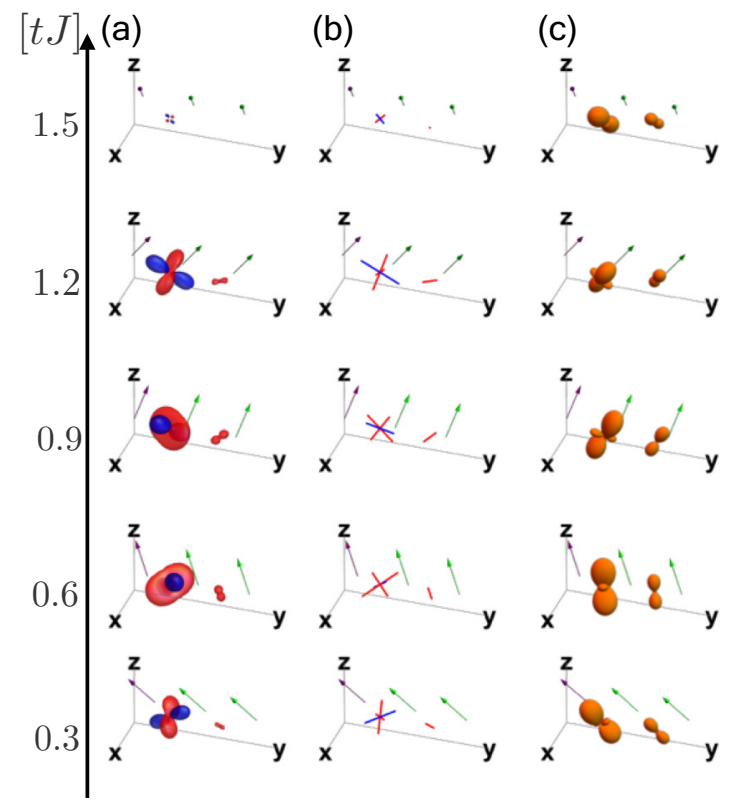

FIG. 8. Comparing visualization methods for two-spin correlations, using (a) CMVs and (b) eigenvector plots with eigenvectors sized according to the corresponding eigenvalue and colored according to the sign; blue and red indicate negative and positive correlations. (c) Wigner functions of the two-spin subsystem.

manners: (1) plotting the eigenvectors and eigenvalues of the correlation matrices and (2) plotting Wigner functions associated with the two-spin subsystem of the many-body system. Examples of these visualizations are shown in Figs. 8(b) and 8(c), respectively. In the Supplemental Material [80], we provide the movies depicting the Ising dynamics using the eigenvector picture and the Wigner plots.

The first method, associating the correlation matrix given by Eq. (3) with geometrical objects by plotting its eigenvectors and eigenvalues, is illustrated in Fig. 8(b). Specifically, to visualize the set of eigenvalues $\lambda_{\alpha}$ and normalized eigenvectors $\vec{v}_{\alpha}$, we draw a rod centered at the origin along the direction of $\vec{v}_{\alpha}$ with length $\left|\lambda_{\alpha}\right|$, and colored according to the sign of $\lambda_{\alpha}$. A rod is the most natural geometrical object to associate with an eigenvector since an eigenvector remains an eigenvector upon inverting its sign. These figures closely parallel the CMVs, as expected from the discussion of Sec. III. In fact, if one imagines fattening out the rods in these figures, they would look like the CMVs. Hence, the differences are almost entirely aesthetic.

The second method, plotting the Wigner functions of the two-spin subsystem whose correlations are being considered, is illustrated in Fig. 8(c). These visualizations merit more discussion, as they are less trivially related to the CMVs. Let us first look at how this visualization method is defined, and then its main features.

To obtain the Wigner functions, we expand the reduced density operator for the two spin-1/2s whose correlation is being considered as

$$
\boldsymbol{\rho}=\sum_{k=0}^{2 j} \sum_{q=-k}^{k} \rho_{k q} \mathbf{T}_{k q}
$$


where $j=1$ for two spin- $1 / 2 \mathrm{~s}, \mathbf{T}_{k q}$ is the multipole operator defined as

$$
\begin{aligned}
\mathbf{T}_{k q}= & \sum_{m=-j}^{j} \sum_{m^{\prime}=-j}^{j}(-1)^{j-m} \sqrt{2 k+1} \\
& \times\left(\begin{array}{ccc}
j & k & j \\
-m & q & m^{\prime}
\end{array}\right)|j m\rangle\left\langle j m^{\prime}\right|,
\end{aligned}
$$

$|j m\rangle$ are the Dicke eigenstates of the total angular momentum operator for the two-spin system, and $\left(\begin{array}{ccc}j_{1} & j_{2} & j_{3} \\ m_{1} & m_{2} & m_{3}\end{array}\right)$ is the $3 j$ symbol. This implicitly defines the $\rho_{k q}$. Following Ref. [53], the corresponding Wigner function is

$$
W(\theta, \phi)=\sum_{k=0}^{2 j} \sum_{q=-k}^{k} Y_{k q}(\theta, \phi) \rho_{k q},
$$

where $Y_{k q}(\theta, \phi)$ are the spherical harmonics. To produce the plots in Fig. 8(c), we make spherical plots of the Wigner function $W(\theta, \phi)$ where, for each value of $\theta, \phi$, the radius of the figure is given as $W(\theta, \phi)$. We also plot only the $k=0$ and $k=2$ components of $W(\theta, \phi)$ in Fig. 8(c) since the $k=1$ information is already provided by the Bloch vectors. For simplicity, we only show the absolute value of $W(\theta, \phi)$, but in general $W(\theta, \phi)$ is a complex number.
Most importantly, we see that the dynamics of the Wigner function, just as for the CMVs, uncovers behavior in this many-body system that is simple to describe. Roughly, the Wigner function rotates at a constant rate around a fixed axis, while its shape changes gradually. This parallels the behavior of the CMVs. The shape of the Wigner function is somewhat different-it is a clover, but oriented differently than the CMVs. There is no reason these shapes should coincide, as they represent the state in different ways. Nevertheless, despite some differences in how they encode the correlations, this method is also capable of yielding the primary result of this paper, revealing a previously hidden geometric structure in manybody systems, just as well as the CMVs (and eigenvector plots).

Although the choice of visualization method is thus somewhat a matter of taste, the CMVs do enjoy some advantages. For one, they are somewhat easier to implement given the correlation functions, requiring one only to plot level sets of the quadratic functions defined by Eqs. (4) and (5). Moreover, if one is particularly interested in the diagonal correlations $\left\langle\left(\sigma_{i} \cdot \mathbf{e}\right)\left(\boldsymbol{\sigma}_{j} \cdot \mathbf{e}\right)\right\rangle-\left\langle\boldsymbol{\sigma}_{i} \cdot \mathbf{e}\right\rangle\left\langle\boldsymbol{\sigma}_{j} \cdot \mathbf{e}\right\rangle$, these are the most straightforward to read off from the CMVs: the size of the CMV along direction $\mathbf{e}$ is roughly proportional to the size of this correlation, as shown in Eq. (8). Perhaps the most important advantage, however, is a purely practical one: our experience is that it is easier to determine the orientation from the CMVs than the eigenvector plots, as the three-dimensional shape supplies more visual cues.
[1] S. J. Maddox, G. Efstathiou, W. J. Sutherland, and J. Loveday, Mon. Not. R. Astron. Soc. 242, 43P (1990).

[2] J. Keeler, Understanding NMR Spectroscopy (Wiley, New York, 2005).

[3] N. F. Rouge, K. J. Wilkinson, and J. Buffle, J. Phys. Chem. B 110, 20133 (2006).

[4] R. Horodecki, P. Horodecki, and M. Horodecki, Phys. Lett. A 200, 340 (1995).

[5] E. Brüning, H. Mäkelä, A. Messina, and F. Petruccione, J. Mod. Opt. 59, 1 (2012).

[6] J. I. de Vicente, J. Phys. A: Math. Theor. 41, 065309 (2008).

[7] J. I. de Vicente, C. Spee, and B. Kraus, Phys. Rev. Lett. 111, 110502 (2013).

[8] N. Linden, S. Popescu, and A. Sudbery, Phys. Rev. Lett. 83, 243 (1999).

[9] A. Laraoui, F. Dolde, C. Burk, F. Reinhard, J. Wrachtrup, and C. A. Meriles, Nat. Commun. 4, 1651 (2013).

[10] H. Strobel, W. Muessel, D. Linnemann, T. Zibold, D. B. Hume, L. Pezzè, A. Smerzi, and M. K. Oberthaler, Science 345, 424 (2014).

[11] T. Aaltonen et al. (CDF Collaboration), Phys. Rev. D 83, 031104 (2011).

[12] G. Mahlon and S. J. Parke, Phys. Rev. D 81, 074024 (2010).

[13] S. Sachdev, Quantum Phase Transitions, 2nd ed. (Cambridge University Press, Cambridge, 2011).

[14] E. Fradkin, Field Theories of Condensed Matter Physics, 2nd ed. (Cambridge University Press, Cambridge, 2013).

[15] C. J. Stevenson and J. Kyriakidis, Phys. Rev. B 83, 115306 (2011).
[16] N. Navon, S. Nascimbène, F. Chevy, and C. Salomon, Science 328, 729 (2010).

[17] N. Cooper, Adv. Phys. 57, 539 (2008).

[18] S. Pielawa, T. Kitagawa, E. Berg, and S. Sachdev, Phys. Rev. B 83, 205135 (2011).

[19] L. D. Carr, D. DeMille, R. V. Krems, and J. Ye, New J. Phys. 11, 055049 (2009).

[20] L. Sanchez-Palencia and M. Lewenstein, Nat. Phys. 6, 87 (2010).

[21] S. Trotzky, Y.-A. Chen, A. Flesch, I. P. McCulloch, U. Schllwöck, J. Eisert, and I. Bloch, Nat. Phys. 8, 325 (2012).

[22] M. Endres, M. Cheneau, T. Fukuhara, C. Weitenberg, P. Schauß, C. Gross, L. Mazza, M. C. Bañuls, L. Pollet, I. Bloch, and S. Kuhr, Appl. Phys. B 113, 27 (2013).

[23] P. Schauß, J. Zeiher, T. Fukuhara, S. Hild, M. Cheneau, T. Macrì, T. Pohl, I. Bloch, and C. Gross, Science 347, 1455 (2015).

[24] T. Vekua, A. Honecker, H.-J. Mikeska, and F. HeidrichMeisner, Phys. Rev. B 76, 174420 (2007).

[25] T.-L. Dao, C. Kollath, I. Carusotto, and M. Köhl, Phys. Rev. A 81, 043626 (2010).

[26] J. Zeiher, R. van Bijnen, P. Schausz, S. Hild, J.-y. Choi, T. Pohl, I. Bloch, and C. Gross, Nat. Phys. 12, 1095 (2016).

[27] H. Weimer, R. Löw, T. Pfau, and H. P. Büchler, Phys. Rev. Lett. 101, 250601 (2008).

[28] H. Labuhn, D. Barredo, S. Ravets, S. de Léséleuc, T. Macrì, T. Lahaye, and A. Browaeys, Nature (London) 534, 667 (2016). 
[29] R. Mukherjee, T. C. Killian, and K. R. A. Hazzard, Phys. Rev. A 94, 053422 (2016).

[30] A. V. Gorshkov, S. R. Manmana, G. Chen, E. Demler, M. D. Lukin, and A. M. Rey, Phys. Rev. A 84, 033619 (2011).

[31] S. R. Manmana, E. M. Stoudenmire, K. R. A. Hazzard, A. M. Rey, and A. V. Gorshkov, Phys. Rev. B 87, 081106 (2013).

[32] B. Yan, S. A. Moses, B. Gadway, J. P. Covey, K. R. A. Hazzard, A. M. Rey, D. S. Jin, and J. Ye, Nature (London) 501, 521 (2013).

[33] R. Islam, C. Senko, W. C. Campbell, S. Korenblit, J. Smith, A. Lee, E. E. Edwards, C.-C. J. Wang, J. K. Freericks, and C. Monroe, Science 340, 583 (2013).

[34] K. Kim, S. Korenblit, R. Islam, E. E. Edwards, M.-S. Chang, C. Noh, H. Carmichael, G.-D. Lin, L.-M. Duan, C. C. J. Wang, J. K. Freericks, and C. Monroe, New J. Phys. 13, 105003 (2011).

[35] R. Blatt and C. F. Roos, Nat. Phys. 8, 277 (2012).

[36] J. W. Britton, B. C. Sawyer, A. C. Keith, C.-C. J. Wang, J. K. Freericks, H. Uys, M. J. Biercuk, and J. J. Bollinger, Nature 484, 489 (2012).

[37] J. G. Bohnet, B. C. Sawyer, J. W. Britton, M. L. Wall, A. M. Rey, M. Foss-Feig, and J. J. Bollinger, Science 352, 1297 (2016).

[38] R. P. Feynman, F. L. Vernon, and R. W. Hellwarth, J. Appl. Phys. 28, 49 (1957).

[39] G. Kimura, Phys. Lett. A 314, 339 (2003).

[40] M. S. Byrd and N. Khaneja, Phys. Rev. A 68, 062322 (2003).

[41] L. Jakóbczyk and M. Siennicki, Phys. Lett. A 286, 383 (2001).

[42] T. Tilma, M. Byrd, and E. C. G. Sudarshan, J. Phys. A 35, 10445 (2002).

[43] R. A. Bertlmann and P. Krammer, J. Phys. A 41, 235303 (2008).

[44] O. Giraud, D. Braun, D. Baguette, T. Bastin, and J. Martin, Phys. Rev. Lett. 114, 080401 (2015).

[45] S. Jevtic, M. Pusey, D. Jennings, and T. Rudolph, Phys. Rev. Lett. 113, 020402 (2014).

[46] C. F. Dunk1, P. Gawron, J. A. Holbrook, J. A. Miszczak, Z. Puchala, and K. Zyczkowski, J. Phys. A 44, 335301 (2011).

[47] P. Kurzynski, A. Kolodziejski, W. Laskowski, and M. Markiewicz, Phys. Rev. A 93, 062126 (2016).

[48] O. Sørensen, G. Eich, M. Levitt, G. Bodenhausen, and R. Ernst, Prog. Nucl. Magn. Reson. Spectrosc. 16, 163 (1984).

[49] T. Halstead, P. Osment, and B. Sanctuary, J. Magn. Reson. 60, 382 (1984).

[50] D. G. Donne and D. G. Gorenstein, Concepts Magn. Reson. 9, 95 (1997).

[51] D. J. Philp and P. W. Kuchel, Concepts Magn. Reson. Part A 25A, 40 (2005).

[52] S. T. Merkel, P. S. Jessen, and I. H. Deutsch, Phys. Rev. A 78, 023404 (2008).

[53] J. P. Dowling, G. S. Agarwal, and W. P. Schleich, Phys. Rev. A 49, 4101 (1994).

[54] D. Harland, M. J. Everitt, K. Nemoto, T. Tilma, and T. P. Spiller, Phys. Rev. A 86, 062117 (2012).

[55] O. Gamel, Phys. Rev. A 93, 062320 (2016).

[56] A. Garon, R. Zeier, and S. J. Glaser, Phys. Rev. A 91, 042122 (2015).

[57] http://www.glasersystems.de/ (unpublished).

[58] D. Leiner, R. Zeier, and S. J. Glaser, Phys. Rev. A 96, 063413 (2017).

[59] R. P. Rundle, P. W. Mills, T. Tilma, J. H. Samson, and M. J. Everitt, Phys. Rev. A 96, 022117 (2017).

[60] http://algassert.com/post/1716. (unpublished)
[61] E. Baake, M. Baake, and H. Wagner, Phys. Rev. Lett. 78, 559 (1997).

[62] M. Gärttner, J. G. Bohnet, A. Safavi-Naini, M. L. Wall, J. J. Bollinger, and A. M. Rey, Nat. Phys. 13, 781 (2017).

[63] A. V. Gorshkov, S. R. Manmana, G. Chen, J. Ye, E. Demler, M. D. Lukin, and A. M. Rey, Phys. Rev. Lett. 107, 115301 (2011).

[64] R. Barnett, D. Petrov, M. Lukin, and E. Demler, Phys. Rev. Lett. 96, 190401 (2006).

[65] A. Micheli, G. K. Brennen, and P. Zoller, Nat. Phys. 2, 341 (2006).

[66] K. R. A. Hazzard, B. Gadway, M. Foss-Feig, B. Yan, S. A. Moses, J. P. Covey, N. Y. Yao, M. D. Lukin, J. Ye, D. S. Jin, and A. M. Rey, Phys. Rev. Lett. 113, 195302 (2014).

[67] K. R. A. Hazzard, S. R. Manmana, M. Foss-Feig, and A. M. Rey, Phys. Rev. Lett. 110, 075301 (2013).

[68] A. de Paz, A. Sharma, A. Chotia, E. Maréchal, J. H. Huckans, P. Pedri, L. Santos, O. Gorceix, L. Vernac, and B. Laburthe-Tolra, Phys. Rev. Lett. 111, 185305 (2013).

[69] A. de Paz, P. Pedri, A. Sharma, M. Efremov, B. Naylor, O. Gorceix, E. Maréchal, L. Vernac, and B. Laburthe-Tolra, Phys. Rev. A 93, 021603 (2016).

[70] N. Takei, C. Sommer, C. Genes, G. Pupillo, H. Goto, K. Koyasu, H. Chiba, M. Weidemüller, and K. Ohmori, Nat. Commun. 7, 13449 (2016).

[71] R. Löw, H. Weimer, U. Krohn, R. Heidemann, V. Bendkowsky, B. Butscher, H. P. Büchler, and T. Pfau, Phys. Rev. A 80, 033422 (2009).

[72] H. Weimer, M. Müller, I. Lesanovsky, P. Zoller, and H. P. Büchler, Nat. Phys. 6, 382 (2010).

[73] L. I. R. Gil, R. Mukherjee, E. M. Bridge, M. P. A. Jones, and T. Pohl, Phys. Rev. Lett. 112, 103601 (2014).

[74] S. Sachdev, K. Sengupta, and S. M. Girvin, Phys. Rev. B 66, 075128 (2002).

[75] F. Meinert, M. J. Mark, E. Kirilov, K. Lauber, P. Weinmann, M. Gröbner, A. J. Daley, and H.-C. Nägerl, Science 344, 1259 (2014).

[76] K. R. A. Hazzard, M. van den Worm, M. Foss-Feig, S. R. Manmana, E. G. Dalla Torre, T. Pfau, M. Kastner, and A. M. Rey, Phys. Rev. A 90, 063622 (2014).

[77] M. Knap, A. Kantian, T. Giamarchi, I. Bloch, M. D. Lukin, and E. Demler, Phys. Rev. Lett. 111, 147205 (2013).

[78] M. Kastner, Phys. Rev. Lett. 106, 130601 (2011).

[79] M. van den Worm, B. C. Sawyer, J. J. Bollinger, and M. Kastner, New J. Phys. 15, 083007 (2013).

[80] See Supplemental Material at http://link.aps.org/supplemental/ 10.1103/PhysRevA.97.043606 for movies of the dynamics of CMV for the Ising and Fermi-Hubbard models.

[81] M. Foss-Feig, K. R. A. Hazzard, J. J. Bollinger, and A. M. Rey, Phys. Rev. A 87, 042101 (2013).

[82] M. Foss-Feig, K. R. A. Hazzard, J. J. Bollinger, A. M. Rey, and C. W. Clark, New J. Phys. 15, 113008 (2013).

[83] M. Gluza, C. Krumnow, M. Friesdorf, C. Gogolin, and J. Eisert, Phys. Rev. Lett. 117, 190602 (2016).

[84] I. G. White, R. G. Hulet, and K. R. A. Hazzard, arXiv:1612.05671.

[85] P. Barmettler, M. Punk, V. Gritsev, E. Demler, and E. Altman, Phys. Rev. Lett. 102, 130603 (2009).

[86] D. Greif, G. Jotzu, M. Messer, R. Desbuquois, and T. Esslinger, Phys. Rev. Lett. 115, 260401 (2015). 
[87] C. J. M. Mathy, D. A. Huse, and R. G. Hulet, Phys. Rev. A 86, 023606 (2012).

[88] P. M. Duarte, R. A. Hart, T.-L. Yang, X. Liu, T. Paiva, E. Khatami, R. T. Scalettar, N. Trivedi, and R. G. Hulet, Phys. Rev. Lett. 114, 070403 (2015).

[89] R. A. Hart, P. M. Duarte, T.-L. Yang, X. Liu, T. Paiva, E. Khatami, R. T. Scalettar, N. Trivedi, D. A. Huse, and R. G. Hulet, Nature (London) 519, 211 (2015).

[90] C. Hofrichter, L. Riegger, F. Scazza, M. Höfer, D. Rio Fernandes, I. Bloch, and S. Fölling, Phys. Rev. X 6, 021030 (2016).

[91] S. Taie, R. Yamazaki, S. Sugawa, and Y. Takahashi, Nat. Phys. 8, 825 (2012).

[92] N. Strohmaier, D. Greif, R. Jördens, L. Tarruell, H. Moritz, T. Esslinger, R. Sensarma, D. Pekker, E. Altman, and E. Demler, Phys. Rev. Lett. 104, 080401 (2010).

[93] J. Simon, W. S. Bakr, R. Ma, M. E. Tai, P. M. Preiss, and M. Greiner, Nature (London) 472, 307 (2011).

[94] B. Zimmermann, T. Müller, J. Meineke, T. Esslinger, and H. Moritz, New J. Phys. 13, 043007 (2011).

[95] C. Sanner, E. J. Su, A. Keshet, W. Huang, J. Gillen, R. Gommers, and W. Ketterle, Phys. Rev. Lett. 106, 010402 (2011).

[96] T. Müller, B. Zimmermann, J. Meineke, J.-P. Brantut, T. Esslinger, and H. Moritz, Phys. Rev. Lett. 105, 040401 (2010).

[97] R. Jordens, N. Strohmaier, K. Gunter, H. Moritz, and T. Esslinger, Nature (London) 455, 204 (2008).

[98] M. F. Parsons, F. Huber, A. Mazurenko, C. S. Chiu, W. Setiawan, K. Wooley-Brown, S. Blatt, and M. Greiner, Phys. Rev. Lett. 114, 213002 (2015).

[99] D. Greif, M. F. Parsons, A. Mazurenko, C. S. Chiu, S. Blatt, F. Huber, G. Ji, and M. Greiner, Science 351, 953 (2016).

[100] L. W. Cheuk, M. A. Nichols, K. R. Lawrence, M. Okan, H. Zhang, E. Khatami, N. Trivedi, T. Paiva, M. Rigol, and M. W. Zwierlein, Science 353, 1260 (2016).

[101] D. Greif, T. Uehlinger, G. Jotzu, L. Tarruell, and T. Esslinger, Science 340, 1307 (2013).
[102] D. Greif, L. Tarruell, T. Uehlinger, R. Jördens, and T. Esslinger, Phys. Rev. Lett. 106, 145302 (2011).

[103] M. Boll, T. A. Hilker, G. Salomon, A. Omran, J. Nespolo, L. Pollet, I. Bloch, and C. Gross, Science 353, 1257 (2016).

[104] D. Schrader, I. Dotsenko, M. Khudaverdyan, Y. Miroshnychenko, A. Rauschenbeutel, and D. Meschede, Phys. Rev. Lett. 93, 150501 (2004).

[105] P. J. Lee, M. Anderlini, B. L. Brown, J. Sebby-Strabley, W. D. Phillips, and J. V. Porto, Phys. Rev. Lett. 99, 020402 (2007).

[106] J. Sebby-Strabley, M. Anderlini, P. S. Jessen, and J. V. Porto, Phys. Rev. A 73, 033605 (2006).

[107] S. Trotzky, P. Cheinet, S. Fölling, M. Feld, U. Schnorrberger, A. M. Rey, A. Polkovnikov, E. A. Demler, M. D. Lukin, and I. Bloch, Science 319, 295 (2008).

[108] Y.-A. Chen, S. Nascimbène, M. Aidelsburger, M. Atala, S. Trotzky, and I. Bloch, Phys. Rev. Lett. 107, 210405 (2011).

[109] F. Gerbier, A. Widera, S. Fölling, O. Mandel, and I. Bloch, Phys. Rev. A 73, 041602 (2006).

[110] A. Widera, F. Gerbier, S. Fölling, T. Gericke, O. Mandel, and I. Bloch, Phys. Rev. Lett. 95, 190405 (2005).

[111] E. H. Lieb and D. W. Robinson, Commun. Math. Phys. 28, 251 (1972).

[112] S. Bravyi, M. B. Hastings, and F. Verstraete, Phys. Rev. Lett. 97, 050401 (2006).

[113] M. Cheneau, P. Barmettler, D. Poletti, M. Endres, P. Schausz, T. Fukuhara, C. Gross, I. Bloch, C. Kollath, and S. Kuhr, Nature (London) 481, 484 (2012).

[114] P. Richerme, Z.-X. Gong, A. Lee, C. Senko, J. Smith, M. FossFeig, S. Michalakis, A. V. Gorshkov, and C. Monroe, Nature (London) 511, 198 (2014).

[115] P. Jurcevic, B. P. Lanyon, P. Hauke, C. Hempel, P. Zoller, R. Blatt, and C. F. Roos, Nature (London) 511, 202 (2014).

[116] P. Pfeuty, Ann. Phys. 57, 79 (1970).

[117] M. A. Valdez, D. Jaschke, D. L. Vargas, and L. D. Carr, Phys. Rev. Lett. 119, 225301 (2017). 